\title{
ON INVARIANTS OF RANDOM PLANAR ENDOMORPHISMS
}

\author{
TEIMURAZ ALIASHVILI \\ Georgian Technical University, Chair of Mathematics No. 99 \\ 77, Kostava str., Tbilisi, Georgia \\ E-mail: aliashvili@mail.ge
}

\begin{abstract}
We estimate the expected value of the gradient degree of certain Gaussian random polynomials in two variables and discuss its relations with some other numerical invariants of random polynomials.
\end{abstract}

Introduction. We deal with random endomorphisms of the plane defined by two random polynomials in two variables and estimate the expected value of the topological degree of such endomorphisms. As usual a polynomial is identified with the collection of its coefficients so a random polynomial is defined by a given distribution of its coefficients. In particular, if the vector of coefficients is a random vector with multidimensional Gaussian (normal) distribution then we speak of a Gaussian random polynomial and we only consider such random polynomials. This setting is quite natural and closely related to important problems of mathematics and physics [10], [6], [5], [7].

For random polynomials in one variable, a fundamental problem is to compute the expected number of real roots, i.e., the mean value (expectation) of the random variable defined as the number of real roots of random polynomial with a given distribution of coefficients. In certain cases this problem was solved in the seminal paper of M. Kac [10]. In particular, M. Kac found the expected number of real roots for random polynomial of fixed algebraic degree $m$ with the coefficients given by independent standard Gaussian random variables [10]. Nowadays there exists vast literature devoted to random polynomials in one variable and the expected number of real roots is known for many classes of random polynomials $[6]$.

Similar problems can be formulated for random polynomials in several variables and random polynomial mappings defined by systems of such polynomials. In particular, by analogy with the one-dimensional case it is natural to consider a random polynomial endomorphism of $\mathbb{R}^{n}$ defined by $n$ random polynomials in $n$ variables with fixed algebraic

2000 Mathematics Subject Classification: 14P25, 60D05.

The paper is in final form and no version of it will be published elsewhere. 
multidegree $M=\left(m_{1}, \ldots, m_{n}\right)$ and compute the expected number of preimages of the origin as a function of $n$ and $m_{i}$.

In other words, this is a question about the expected number of real roots of the corresponding $(n \times n)$-system of random polynomial equations. Obviously, one can regard this number as a certain numerical invariant of the given random polynomial endomorphism of $\mathbb{R}^{n}$ which may be interpreted as its expected geometric multiplicity. Recently, M. Shub and S. Smale succeeded to compute this invariant in arbitrary dimension for certain special distributions of coefficients [19], which gives a wide generalization of the mentioned classical results of M. Kac. These and other recent developments in the spirit of [19] are summarized in [8].

Despite this progress many natural problems remain uninvestigated even for random endomorphisms of the plane $\mathbb{R}^{2}$ which we call random planar endomorphisms (or random plends). Here we consider one problem of topological nature which was formulated by G. Khimshiashvili in the context of algebraic methods for computing topological invariants [12] and investigated in [12], [13], [14] (cf. also [17]).

Notice that a Gaussian random planar endomorphism is almost certainly (i.e., with probability one) proper and stable in the sense of Whitney [4], in which case we say that it is typical. As is well known, for a typical planar endomorphism one can introduce several useful numerical characteristics of its geometric behaviour such as the topological (mapping) degree [12], the number of cusps [4], and the number of complex points [15]. Hence one can consider the expected values of these characteristics and obtain in this way numerical invariants of a given random planar endomorphism. Following the strategy suggested in [12], we study the first invariant using the techniques described in [12] and then briefly outline how it can be used for estimating the two other invariants.

More precisely, we find the expected value of the topological degree of a random planar endomorphism defined by the gradient of a random polynomial with a certain rotation invariant Gaussian distribution of coefficients introduced in [19] (cf. Theorem 3.1 below). Using the mapping degree formula for the Euler characteristic established in [11], it can be shown that our result agrees with the results about the average Euler characteristic of random hypersurfaces obtained in [17]. If all coefficients of a random polynomial are standard normals, we indicate the asymptotic of the expected value of gradient degree as the algebraic degree of the random polynomial tends to infinity (Theorem 3.2).

Obviously, topological aspects of random polynomial mappings are too vast to be satisfactorily covered in a single paper. Our aim was just to make first few steps in the direction suggested in [12].

Acknowledgements. The author's work on this paper was supported by INTAS grant 00-259. The author is grateful to G. Khimshiashvili for suggesting the topic and numerous useful discussions.

1. Gaussian random polynomials. As was already mentioned, we deal with Gaussian random polynomials, which means that their coefficients are real random variables and have multivariate normal distribution. The term "random polynomial" always refers 
to this situation. To be more precise, we reproduce some concepts and notation from probability theory. All necessary background can be found in [18].

Recall that a (one-dimensional) Gaussian (normal) random variable $\xi$ is defined as a real-valued random variable with Gaussian (normal) density

$$
f_{\xi}(x)=\frac{1}{\sqrt{2 \pi} \sigma} \exp \left(-\frac{(x-a)^{2}}{2 \sigma^{2}}\right),
$$

where $\sigma>0,-\infty<a<+\infty$. Parameters $a$ and $\sigma$ determine its expectation and variance:

$$
a=E \xi, \sigma^{2}=D \xi \text {. }
$$

If $a=0$, we speak of a central normal distribution. If $a=0$ and $\sigma=1$ one obtains the standard normal distribution

$$
\Phi(x)=\frac{1}{\sqrt{2 \pi}} \cdot \int_{-\infty}^{x} e^{-u^{2} / 2} d u .
$$

Recall also that, for a pair of random variables $(\xi, \eta)$, the number

$$
\operatorname{cov}(\xi, \eta)=E[(\xi-E \xi) \cdot(\eta-E \eta)]
$$

is called the covariation of $\xi$ and $\eta$. If $\operatorname{cov}(\xi, \eta)=0$, then $\xi$ and $\eta$ are called non-correlated, in particular (stochastically) independent random variables are non-correlated [18]. The variance $D \xi$ is defined as $\operatorname{cov}(\xi, \xi)=D \xi$.

Recall finally that a multidimensional central normal distribution $\Xi$ is defined as a distribution with density

where

$$
f_{\Xi}(x)=\frac{\sqrt{|A|}}{(2 \pi)^{n / 2}} \cdot e^{-Q(x) / 2},
$$

$$
Q(x)=x A x^{T}=\sum_{i, j=1}^{n} a_{i j} x_{i} x_{j},
$$

and $|A|$ denotes the determinant of a positive definite matrix $A=\left(a_{i j}\right)$. The word central indicates again that $E \Xi=0$ (sometimes one says that this is a distribution with zero mean [8]). The matrix $A$ is called the covariance matrix of the above distribution because its elements are given by pairwise covariances of the components of distribution $\Xi$.

Let $P$ be a Gaussian random polynomial on $\mathbb{R}^{n}$ in the above sense. Then it can be written as

$$
P(x)=\sum_{\alpha} \xi_{\alpha} x^{\alpha}
$$

$\alpha=\left(\alpha_{1}, \alpha_{2}, \ldots, \alpha_{n}\right), x=\left(x_{1}, x_{2}, \ldots, x_{n}\right), x^{\alpha}=x_{1}^{\alpha_{1}} \cdot x_{2}^{\alpha_{2}} \cdot \ldots \cdot x_{n}^{\alpha_{n}}$, where $\xi_{\alpha}$ have multidimensional normal distribution. To specify this distribution it is sufficient to give its covariance matrix explicitly. Denote by $m$ the algebraic degree of $P$.

EXAMPLE 1.1. Let all coefficients $\xi_{\alpha}$ be i.i.d. (independent identically distributed) standard normals. Then $P$ is called a standard Gaussian random polynomial in $n$ variables of algebraic degree $m$.

For polynomials in one variable, such type of random polynomial is quite natural and standard and there exist a lot of results about numerical invariants of standard Gaussian 
polynomials in one variable [6]. However, it turned out that in the case of several variables such polynomials are sufficiently difficult to work with, so for us especially important is the following example of Gaussian random polynomial introduced in [19].

Example $1.2([19])$. Denote by $H_{m}\left(\mathbb{R}^{n+1}\right)$ the set of all homogeneous polynomials of algebraic degree $m$ ( $m$-forms) on $\mathbb{R}^{n+1}$ and consider a Gaussian random homogeneous polynomial $f$ from $H_{m}\left(\mathbb{R}^{n+1}\right)$ having the form

$$
F(x)=\sum_{m_{0}+m_{1}+\ldots+m_{n}=m} F_{m_{0}, m_{1}, \ldots, m_{n}} x_{0}^{m_{0}} x_{1}^{m_{1}} \ldots x_{n}^{m_{n}}, \quad x \in \mathbb{R}^{n+1},
$$

where $F_{m_{0}, \ldots, m_{n}}$ are independent normal random variables with zero mean and variances

$$
E F_{m_{0}, \ldots, m_{n}}^{2}=\frac{m !}{m_{o} ! \ldots m_{n} !} .
$$

We call it a standard Gaussian random $m$-form.

It can be verified that such a random polynomial is invariant with respect to the natural action of the group $O(n+1)$ on $H_{m}\left(\mathbb{R}^{n+1}\right)$ in which case it is said to be rotation invariant. As was shown in [17], an important characteristic of a rotation invariant Gaussian random polynomial is given by the so-called parameter $r$ which is defined by the formula

$$
r=\frac{E\left(\frac{\partial F}{\partial x_{0}}(e)\right)^{2}}{E F(e)^{2}}
$$

where $e=(1,0, \ldots, 0) \in S^{n}$ is a chosen point. It is possible to check by a direct computation that in the above example we get $r=m$.

There is one more type of random polynomial which is relevant for our purposes. It was introduced in [17] and can be described as follows.

EXAMPLE $1.3([17])$. Introduce a scalar product on $H_{m}\left(\mathbb{R}^{n+1}\right)$ by the formula

$$
\left(f, f^{\prime}\right)=\int_{x \in S^{n}} f(x) f^{\prime}(x) d x, \quad f, f^{\prime} \in H_{m}\left(\mathbb{R}^{n+1}\right) .
$$

A Gaussian random $m$-form $F$ is called satisfactory if

$$
E\left((f, F)\left(f^{\prime}, F\right)\right)=\left(f, f^{\prime}\right), \quad f, f^{\prime} \in H_{m}\left(\mathbb{R}^{n+1}\right) .
$$

According to [17] such a random polynomial is rotation invariant and its parameter $r$ is equal to

$$
\frac{m(m+n+1)}{n+2}
$$

Consider now a general (i.e. not necessarily homogeneous) Gaussian random polynomial on $\mathbb{R}^{n+1}$ of algebraic degree $N$ such that each of its homogeneous components of degree $m \leq N$ has standard Gaussian distribution specified in Example 1.1. In such situation we speak of a standard random polynomial of algebraic degree $N$. This is precisely the type of random polynomial we wish to consider. There are (at least) two main reasons for such choice: first, such polynomials and their numerical invariants appeared useful in physics [7], [5], and, second, for systems of random polynomials of such type M. Shub and S. Smale [19] obtained simple explicit formulae for the expected number of 
real roots, which enable one to obtain various results about numerical invariants of such polynomials.

One can analogously define a (non-homogeneous) satisfactory random polynomial of algebraic degree $N$. For such polynomials, one can also obtain some useful results about their numerical invariants.

2. Expected number of real roots. Our discussion in the next section relies on some results about the expected number of real roots of random polynomials in one variable. So we reproduce several relevant results from [8]. Let

$$
P(x)=a_{0}+a_{1} x+\ldots+a_{m} x^{m}
$$

be a non-zero polynomial in one variable. Define two (column) vectors

$$
a=\left(a_{0}, a_{1}, \ldots, a_{m}\right)^{T}, \quad V(t)=\left(1, t, t^{2}, \ldots, t^{m}\right)^{T} .
$$

Suppose now that $P$ is a Gaussian random polynomial as above with some fixed distribution of coefficients and denote by $E_{m}$ the expected number of its real roots. Obviously, this number depends on the distribution of vector $a$. A beautiful geometric argument going back to [19] shows that in the case of standard Gaussian polynomial $P$ (actually the same holds if $a_{i}$ are any i.i.d. central normals)

$$
E_{m}=\frac{1}{\pi}|\gamma|
$$

where $\gamma(t) \equiv V(t) /\|V(t)\|$ is the normalized moment curve on the unit sphere in $\mathbb{R}^{m+1}$ and $|\gamma|$ denotes its length [8]. As was shown in [8], this enables one to compute the expected number of real roots for various Gaussian random polynomials in one variable. In particular, for a standard Gaussian polynomial of algebraic degree $n$, one has the famous Kac formula [10].

PROPOSITION 2.1. The expected number of real roots of a standard Gaussian random polynomial of algebraic degree $n$ is

$$
E_{m}=\frac{1}{\pi} \int_{-\infty}^{+\infty} \sqrt{\left.\frac{\partial^{2}}{\partial x \partial y}\left(\log \frac{1-(x y)^{m+1}}{1-x y}\right)\right|_{y=x=t}} d t
$$

This version of Kac formula appeared in [8]. It is standard from many points of view. For example, using it one can write down an asymptotic expansion of $E_{m}$ as $m$ tends to infinity [8]. In particular, it is known that $E_{m}$ grows as $\frac{1}{\pi} \log m$ as $m$ tends to infinity.

As was shown in [19], very elegant and explicit results about $E_{m}$ can be obtained for standard Gaussian polynomial given in Example 1.2. Notice that such random polynomials in one variable have also been studied in the context of some problems of quantum physics [7]. Although this is just a special case of Example 1.2 we describe it explicitly because of its importance.

Let $P(x)=a_{0}+a_{1} x+\ldots+a_{m} x^{m}$ be a random polynomial with the coefficients $a_{i}$ which are independent normal random variables with variances $\left(\begin{array}{c}m \\ i\end{array}\right)$. As was shown in [8], the density of zeros in this case is

$$
\rho(t)=\frac{\sqrt{m}}{\pi\left(1+t^{2}\right)} .
$$


So in this case the density of zeros is given by the Cauchy distribution [18]. Integrating this density one obtains a simple formula for $E_{m}$ which we need in the next section.

Proposition 2.2 ([19]). The expected number of real roots of standard Gaussian random polynomial in one variable of algebraic degree $m$ is equal to $\sqrt{m}$.

Similar results hold for the expected number of real roots of random polynomial systems defined by Gaussian random polynomials. The following example is instructive from the point of view of the planar endomorphisms.

EXAMPLE 2.1. Let $(P, Q)$ be a pair of standard Gaussian random polynomials in two variables of algebraic degree $m$. Then the expected number of their common real roots is equal to $m$.

In other words, in this case one is able to find the exact value of expected geometric multiplicity so it becomes natural to try the same for other numerical invariants of a random plend which were mentioned in the introduction. As is shown in the next section, in some cases one can find or estimate its expected gradient degree.

3. Expected gradient degree in the plane. Let $\mathbb{R}_{2}$ be the ring of real polynomials of two variables. For $P \in \mathbb{R}_{2}$, let $\operatorname{deg} P$ denote its algebraic degree, i.e. the highest order of monomials which appear in $P$. For convenience, we write a polynomial $P$ with $\operatorname{deg} P=m$ in the form

$$
P(x, y)=\sum_{k+l=0}^{m} a_{k l} x^{k} y^{l},
$$

where at least one non-vanishing $a_{k l}$ with $k+l=m$ appears. The leader $P^{*}$ is defined as the sum of monomials of highest order. Obviously it is a non-trivial binary $m$-form.

By taking a pair of random polynomials of the above form (not necessarily with the same distribution of coefficients) one gets a random plend

$$
F=(P, Q): \mathbb{R}^{2} \longrightarrow \mathbb{R}^{2}
$$

with these polynomials as the components. In this way one can define Gaussian random plends of various types.

As is well known, if $F$ is proper then its (global) topological degree $\operatorname{Deg} F$ is welldefined [16]. As one could expect, a random plend almost certainly has several nice properties of which we need at the moment only one. It can be proved applying the same reasoning as was used in [17] to show that a random Gaussian hypersurface is almost certainly smooth.

Proposition 3.1. A Gaussian random plend is proper with probability one.

Actually, the same is true for many other generic properties of plends, such as stability in the sense of Whitney [4], which enables one to introduce the random variable equal to the number of its cusps and consider the expected number of cusps.

Proposition 3.2. A Gaussian random plend is stable with probability one.

For those $\omega$ for which $F(\omega)$ is not proper, we set $\operatorname{Deg} F(\omega)=0$. In this section we are concerned with estimating the expectation $E(\operatorname{Deg} F)$ of random variable $\operatorname{Deg} F$. 
In order to obtain the desired result we need to restrict admissible distributions of coefficients $a_{k l}$. It turns out that an appropriate setting is provided by Example 1.2 in the special case $n=2$. Namely, we start with a random polynomial $P$ of algebraic degree $m$ whose coefficients $a_{k l}$ are independent central Gaussian variables with variances given by "trinomial coefficients"

$$
D a_{k l}=E a_{k l}^{2}=\frac{m !}{k ! l !(m-k-l) !}
$$

and consider a random plend $P^{\prime}$ defined as the gradient of $P$, i.e., the components of $P^{\prime}$ are given by the partial derivatives $P_{x}, P_{y}$. In other words, we deal with the gradient of a standard random polynomial in two variables.

This is obviously a Gaussian random plend and it is easy to write down the covariance matrix of its coefficients. Notice that the components of $P^{\prime}$ are neither independent nor have the identical distribution of coefficients. Such random plends were earlier considered in [14]. As was mentioned in the introduction, they are relevant in some physical problems, which, in our opinion, gives a sufficient motivation for studying them by their own.

THEOREM 3.1. Let $P$ be a standard Gaussian random polynomial in two variables of algebraic degree $m \geq 1$. Then the expectation $E\left(\operatorname{Deg} P^{\prime}\right)$ of the topological degree of its gradient $P^{\prime}$ is equal to $1-\sqrt{m}$.

Proof. First of all, notice that it is sufficient to estimate the average topological degree of the endomorphism $\left(P^{*}\right)^{\prime}$ defined by leaders $P_{x}^{*}, P_{y}^{*}$ which are binary homogeneous $(m-1)$-forms.

Lemma 3.1. $E\left(\operatorname{Deg} P^{\prime}\right)=E\left(\operatorname{Deg}\left(P^{*}\right)^{\prime}\right)$.

Indeed, it is easy to see that almost certainly the components of $F^{*}$ have no common nontrivial roots. As is well known (see, e.g., [16]), in such case there exists an admissible (proper) homotopy between $P^{\prime}$ and $\left(P^{\prime}\right)^{*}$ so their topological degrees coincide almost certainly, which implies the lemma. So it is sufficient to prove the theorem for a homogeneous random polynomial $P^{*}$.

Notice further that the zero set $Z$ of a homogeneous polynomial $P^{*}$ in two variables consists of a system of lines in $\mathbb{R}^{2}$ passing through the origin. Their intersections with the unit circle $S^{1}$ give a finite set of points $Y=Z \cap S^{1}$. These points obviously appear in pairs and those pairs are in a one-to-one correspondence with the real roots of polynomial in one variable $\widehat{P}$ which is obtained from $P^{*}$ by dehomogenization (i.e. we divide $P^{*}(x, y)$ by $y^{m}$ and introduce a new variable $t=\frac{x}{y}$ ). In other words, the number $k$ of points in $Y$ equals $2 r$, where $r$ is the number of real roots of $\widehat{P}$.

At the same time the number $k$ can be obviously interpreted as the Euler characteristic $\chi(Y)$ of the set $Y$. According to [12], the Euler characteristic of the zero-set of homogeneous polynomial $P^{*}$ is related to the mapping degree of its gradient by the formula

$$
\chi(Y)=2\left(1-\operatorname{Deg}\left(P^{*}\right)^{\prime}\right) .
$$

Thus $r=1-\operatorname{Deg}\left(P^{*}\right)^{\prime}$ (cf. [16]). Thus we will be able to compute the expected value of gradient degree if we find the expected value of the random variable equal to $r$. This 
appears possible due to the following observation which is verified by a straightforward check.

LEMMA 3.2. $\widehat{P}$ is a standard Gaussian random polynomial in one variable of algebraic degree $m$.

Notice now that by Proposition 2.2 the expected number of its real roots $E(r)$ is equal to $\sqrt{m}$. So the result follows by taking the expectations of both sides of the above formula and applying Lemma 3.1. The proof is thus completed.

We would like to point out that the above argument shows as well that the expectation of the Euler characteristic of the zero-set of $P$ intersected with the unit circle is equal to $2 \sqrt{m}$. The expectation of Euler characteristic of the projectivized zero-set of Gaussian random homogeneous polynomial with rotation invariant distribution of coefficients was computed in [17]. Applying the formula from [17] in our case one gets that the latter expectation is equal to $\sqrt{m}$, which agrees with our result since the set $Y$ above is the double of the projectivized zero-set.

It is easy to see that the same geometric argument enables one to obtain similar results for Gaussian random polynomials of other types. In particular, arguing in the same way as above and using Proposition 2.1 and the asymptotic of the Kac formula indicated thereafter, one can find the asymptotic of expected gradient degree for standard Gaussian polynomial in two variables.

ThEOREM 3.2. The expected value of the gradient degree of standard Gaussian random polynomial in two variables of algebraic degree $m$ is asymptotically equivalent to $-\frac{1}{\pi} \log m$ as $m$ tends to infinity.

The list of such results could be extended since the expected number of real roots of $\widehat{P}$ can be estimated in many cases. For example, the same argument works for the gradient of satisfactory random polynomial from Example 1.3 but we do not dwell upon the details for the sake of brevity. There is good evidence that similar arguments based on the formulae from [12] may work in higher dimensions but it does not seem proper to go into that in the context of this note.

In the conclusion we wish to say a few words about some other invariants of random plends mentioned in the introduction, namely, the expectations $E(c(F))$ and $E(s(F))$, where $c(F)$ and $s(F)$ are the number of complex points and cusps of $F$, respectively. A natural setting in this context is to consider random plends with fixed algebraic degrees of the components.

Recall that the set of complex points $\mathbb{C}(F)$ of a plend $F$ by definition consists of such points at which tangent plane to its graph $\Gamma_{F} \subset \mathbb{C}^{2} \cong \mathbb{R}^{2} \times \mathbb{R}^{2}$ is a complex line in $\mathbb{C}^{2} \cong \mathbb{R}^{4}[15]$. It can be shown that a Gaussian random plend almost certainly has a finite number $c(F)$ of complex points so it makes sense to consider the expectation $E(c(F))$. From Proposition 3.2 it follows that the same can be said about the number of cusps $s(F)$.

Both these expectations give useful characteristics of a random plend. For example, $E(c(F))$ would give an estimate for the number of analytic discs attached to the graph of $F[15]$ while the expected number of cusps and ombilic points plays certain role in 
M. Berry's approach to the diffraction on Gaussian random surfaces (see [5] and references therein). So it seems really tempting to express these invariants through the algebraic degrees of the components.

A natural first step in this direction is to compute these invariants for the gradient of standard random polynomial as above. The relation of this problem to our results about the expected gradient degree is given by a variety of so-called mapping degree formulae for these invariants [1], [2], [3], [9], [12], [13], [15] which enable one to compute them in terms of the topological degree of effectively constructible gradient plends and estimate them from above in terms of the algebraic degree. However we do not discuss these developments here because in all known applications (cf. [5], [7]) one is interested in exact values of the expectations in question which up to now remain beyond reach.

\section{References}

[1] T. Aliashvili, On the estimation of the root number of a polynomial endomorphism over an $\bar{R}$-field, Soobshch. Akad. Nauk. Gruzii 148 (1993), 32-35.

[2] T. Aliashvili, On the topological degree of a polynomial endomorphism of an affine space, Proc. A. Razmadze Math. Inst. 104 (1994), 11-25 (Russian).

[3] T. Aliashvili, Signature method for counting points in semi-algebraic subsets, Bull. Georgian Acad. Sci. 154 (1996), 34-36.

[4] V. I. Arnol'd, A. N. Varchenko, S. M. Guseřn-Zade, Singularities of Differentiable Maps I, Nauka, Moskva, 1982 (Russian).

[5] M. Berry, Statistics of nodal lines and points in chaotic quantum billiards: perimeter corrections, fluctuations, curvature, J. Phys. A 35 (2002), 3025-3038.

[6] A. T. Bharucha-Reid, M. Sambandham, Random Polynomials, Academic Press, Orlando, 1986.

[7] E. Bogomolny, O. Bohigas, P. Lebœuf, Distribution of roots of random polynomials, Phys. Rev. Lett. 68 (1992), 2726-2729.

[8] A. Edelman, E. Kostlan, How many roots of a random polynomial are real?, Bull. Amer. Math. Soc. (N.S.) 32 (1995), 1-37; Erratum: ibid. 33 (1996), 325.

[9] T. Fukuda, G. Ishikawa, On the number of cusps of stable perturbations of a plane-to-plane singularity, Tokyo J. Math. 10 (1987), 375-384.

[10] M. Kac, On the average number of real roots of a random algebraic equation, Bull. Amer. Math. Soc. 49 (1943), 314-320; correction: ibid., 938.

[11] G. Khimshiashvili, On the local degree of a smooth mapping, Bull. Acad. Sci. Georgian SSR 85 (1977), 309-312 (Russian).

[12] G. Khimshiashvili, Signature formulae for topological invariants, Proc. A. Razmadze Math. Inst. 125 (2001), 1-121.

[13] G. Khimshiashvili, On local invariants of totally real surfaces, Georgian Math. J. 8 (2001), 97-109.

[14] G. Khimshiashvili, A. Ushveridze, On the average topological degree of random polynomials, Bull. Georgian Acad. Sci. 159 (1999), 385-388.

[15] G. Khimshiashvili, E. Wegert, Analytic discs and complex points of surfaces, Bull. Soc. Sci. Lettres Łódź 52 (2002), 147-166.

[16] M. A. Krasnosel'skiľ et al., Vector fields in the plane, Fizmatgiz, Moscow, 1963 (Russian). 
[17] S. S. Podkorytov, The mean value of the Euler characteristic of a random algebraic hypersurface, Algebra i Analiz 11:5 (1999), 185-193 (Russian); English transl.: St. Petersburg Math. J. 11 (2000), 853-860.

[18] A. Shiryaev, Probability, Nauka, Moscow, 1980 (Russian); English transl.: Graduate Texts in Math. 95, Springer, New York, 1984.

[19] M. Shub, S. Smale, Complexity of Bezout's theorem II. Volumes and probabilities, in: Computational Algebraic Geometry (Nice, 1992), Progr. Math. 109, Birkhäuser, Boston, 1993, $267-285$. 